\title{
NOVOS DESAFIOS PARA VELHOS PROBLEMAS: O ENFERMEIRO ESPECIALISTA EM ENFERMAGEM DE REABILITAÇÃO NA PROMOÇÃO DA ACESSIBILIDADE
}

\author{
NuEVOS DESAFÍOS PARA VIEJOS PROBLEMAS: EL ENFERMERO ESPECIALISTA EN ENFERMERIA DE REHABILITACIÓN EN LA \\ PROMOCIÓN DE LA ACCESIBILIDAD
}

\section{NEW CHALLENGES FOR OLD PROBLEMS: THE SPECIALIZED NURSE IN REHABILITATION NURSING ON ACCESSIBILITY PROMOTION}

DOI 10.33194/rper.2019.v2.n2.02.4561 | Submetido 03.03.2019 | Aprovado 28.11.2019

\begin{abstract}
Carina Ferreira da Silva ${ }^{1,4}$; Fábio Daniel Barros de Oliveira ${ }^{2,4}$; Marlene Patrícia Ribeiro ${ }^{2,4}$; Virgínia Maria Pereira Prazeres ${ }^{3,4}$; Olga Maria Pimenta Lopes Ribeiro ${ }^{5,6}$
\end{abstract}

1 - Centro de Reabilitação do Norte; 2 - Centro Hospitalar do Tâmega e Sousa; 3 - Centro Hospitalar Universitário do Porto; 4Escola Superior de Saúde de Santa Maria; 5 - Escola Superior de Enfermagem do Porto; 6 - CINTESIS

\section{RESUMO}

Enquadramento: A acessibilidade constitui um elemento crucial na qualidade de vida das pessoas com mobilidade condicionada, sendo imprescindível para o exercício dos seus direitos. A sua efetividade envolve uma equipa multidisciplinar, que deverá incluir o Enfermeiro Especialista em Enfermagem de Reabilitação, dado que a este compete capacitar a pessoa com mobilidade condicionada para a reinserção e exercício da cidadania.

Objetivos: Refletir sobre os direitos das pessoas com mobilidade condicionada; compreender a intervenção do Enfermeiro Especialista em Enfermagem de Reabilitação na promoção da acessibilidade e na inclusão social.

Principais tópicos em análise: Legislação e planos de promoção da acessibilidade e da inclusão social; e a intervenção do Enfermeiro Especialista em Enfermagem de Reabilitação no âmbito destas problemáticas.

Conclusão: As condições de acessibilidade constituem um dos fatores discriminatórios para as pessoas com mobilidade condicionada. Assim, compete ao Enfermeiro Especialista em Enfermagem de Reabilitação paralelamente com as entidades competentes, encarar esta problemática como uma urgente oportunidade para a mudança.

Palavras-chave: Acessibilidade Arquitetônica; Enfermagem em Reabilitação; Limitação da Mobilidade; Pessoas com Deficiência.

\section{RESUMEN}

Encuadramiento: La accesibilidad es un elemento crucial en la calidad de vida de las personas con movilidad reducida, siendo imprescindible para el ejercicio de los sus derechos. La efectividad implica un equipo multidisciplinario, que deberá incluir el Enfermero Especialista en Enfermería de Rehabilitación, dado que a éste compete capacitar a la persona con movilidad condicionada para la reinserción y ejercicio de la la ciudadanía.

Objetivos: Reflexionar sobre los derechos de las personas con movilidad reducida; comprender la intervención del Enfermero Especialista en Enfermería de Rehabilitación en la promoción de la accesibilidad y la inclusión social.

Principales temas en análisis: legislación y planes de promoción de la accesibilidad y de la inclusión social; y la intervención del Enfermero Especialista en Enfermería de Rehabilitación en el marco de estas problemáticas.

Conclusión: Las condiciones de accesibilidad constituyen uno de los factores discriminatorios para las personas con movilidad reducida. Por lo tanto, compete al Enfermero Especialista en Enfermería de Rehabilitación paralelamente con las autoridades competentes, encarar esta problemática como una urgente oportunidad para el cambio.

Palabras clave: Accesibilidad Arquitectónica; Enfermería en Rehabilitación; Limitación de la Movilidad; Personas con Discapacidad.

\section{ABSTRACT}

Background: Accessibility is a crucial element in the quality of life of disabled people, being indispensable for the exercise of their rights. Its effectiveness involves a multidisciplinary team, which should include the nurse specialist in rehabilitation nursing, since it is incumbent that this has to empower the disabled person for the reintegration and exercise of citizenship.

Objectives: Reflect on the rights of persons with disabilities; to understand the intervention of the nurse specializing in rehabilitation nursing in accessibility promotion and social inclusion. 
Main topics under analysis: legislation and plans to promote accessibility and social inclusion; and the intervention of the nurse specializing in rehabilitation nursing in the context of these problems.

Conclusion: Accessibility conditions constitute one of the discriminatory factors for disabled people. Thus, the nurse specialized in rehabilitation nursing in parallel with the competent authorities, should face this problem as an urgent opportunity for the promotion of a change.

Keywords: Architectural Accessibility; Rehabilitation Nursing; Mobility Limitation; Disabled Persons.

\section{INTRODUÇÃO}

As pessoas com mobilidade condicionada são pessoas que utilizam cadeira de rodas, incapazes de andar ou que não conseguem percorrer grandes distâncias, com dificuldades sensoriais (cegos ou surdos) e aquelas que se apresentam transitoriamente condicionadas (grávidas, crianças e idosos). Estas pessoas confrontamse com barreiras ambientais que são impeditivas de uma participação cívica ativa e integral, sendo obrigação do Estado garantir e assegurar os direitos $\operatorname{destas}^{(1)}$.

A Classificação Internacional de Funcionalidade, Incapacidade e Saúde (CIF) correlaciona a incapacidade com a funcionalidade. Define que as mesmas resultam de uma relação complexa entre a condição de saúde do indivíduo (doenças, perturbações e lesões) e os fatores pessoais (sociais, económicos, literários), com os fatores externos (ambientais) que representam as circunstâncias nas quais o indivíduo vive ${ }^{(2)}$. As pessoas com mobilidade condicionada são confrontadas, diariamente, com a problemática das barreiras arquitetónicas.

De acordo com alguns autores, as barreiras arquitetónicas estão presentes nos diferentes espaços e contextos e constituem obstáculos, dificultando ou impedindo a livre circulação de pessoas que vivenciam uma incapacidade (transitória ou permanente) ${ }^{(3)}$.

As acessibilidades, que vão desde as ajudas técnicas ao acesso dos edifícios, constituem uma condição essencial para o pleno exercício dos direitos. A garantia da autonomia e do acesso a bens e serviços existentes na sociedade para todos, tem sido objetivo da legislação adotada e atualizada bem como dos planos de ação por parte de entidades públicas e privadas, atribuindo maior visibilidade a esta causa com o objetivo de respeitar os direitos das pessoas com mobilidade condicionada e da sociedade inclusiva para todos.

Atualmente existem no mundo mais de um bilião de pessoas que apresentam alguma forma de deficiência, e entre estas, 200 milhões apresentam dificuldades funcionais consideráveis ${ }^{(4)}$. De resto, prevê-se que na Europa, no ano de 2050, 22\% da população tenha menos de vinte anos e cerca de $40 \%$ tenha mais de 65 anos $^{(5)}$. Prevê-se, assim, que exista um aumento da população com mobilidade condicionada de uma forma global.

De acordo com os Censos 2011(6), em Portugal estimase existir $18 \%$ de pessoas com mobilidade condicionada, prevendo-se um aumento da prevalência desta população em $50 \%$ até $2050^{(7)}$.

A promoção e a garantia da plena acessibilidade é essencial à qualidade de vida dos cidadãos e ao exercício dos deus direitos, como membros participantes de uma comunidade regida pelos princípios de uma sociedade democrática, no sentido de assegurar a sua real participação e integração cívica. Assim, a acessibilidade traduz-se em vantagens para todos: permite o exercício pleno da cidadania e participação ativa nos diversos domínios de atividade da sociedade; assegura, ao maior número possível de cidadãos, a possibilidade de viverem integrados na sua comunidade em situações de equidade de oportunidades; contribui para que os espaços e serviços proporcionem condições de segurança e conforto; e assegura menores encargos na autonomia e na maior independência a todos os cidadãos ${ }^{(8)}$.

Tendo em conta as competências do Enfermeiro Especialista em Enfermagem de Reabilitação (EEER), torna-se indiscutível a sua importância na promoção de ambientes inclusivos. Assim, este deve envolver-se na melhoria das condições de acessibilidade, com uma atitude mais proativa nesta área com o sentido de aumentar os ganhos em qualidade de vida das pessoas com mobilidade condicionada. Em parceria com os órgãos autárquicos locais, podem "constituir um binómio de promoção da saúde e da acessibilidade arquitetónica, uma vez que, são os atores políticos de construção de territórios inclusivos, pelo cumprimento de disposições legais, construindo cidades inclusivas para pessoas com mobilidade condicionada" (9).

A eliminação de barreiras arquitetónicas é fundamental para que as pessoas com mobilidade condicionada possam ter acesso a todos os sistemas e serviços da comunidade e assim, gozar dos seus direitos como cidadãos $^{(8)}$.

É neste enquadramento, que se considerou pertinente, refletir sobre o contributo do EEER na promoção da acessibilidade e inclusão social, tendo por base a legislação e as políticas de acessibilidade atuais, bem como os documentos reguladores do exercício profissional e os referenciais teóricos de enfermagem.

Para a concretização deste estudo teórico focamo-nos na legislação, nos programas, nos planos de ação e nas políticas em vigor, nas competências especificas do EEER, nos padrões de qualidade dos cuidados especializados em enfermagem de reabilitação, bem como em publicações que contemplassem esta temática.

Embora o ambiente, enquanto conceito metaparadigmático, seja considerado pelos EEER um componente facilitador ou dificultador da adaptação da pessoa com mobilidade condicionada, a existência de lacunas na intervenção a este nível, motivou a realização deste artigo teórico. $\mathrm{Na}$ sequência da reflexão realizada, para além de evidenciarmos o 
contributo do EEER, é nossa pretensão sensibilizar estes profissionais para uma intervenção sustentada no âmbito desta problemática, determinante na qualidade de vida e no exercício da cidadania da pessoa com mobilidade condicionada.

\section{DESENVOLVIMENTO}

$\mathrm{Na}$ Convenção sobre os Direitos da Pessoa com Deficiência (CDPD) ${ }^{(10)}$, define-se deficiência como um conceito evolutivo que não se circunscreve, apenas, às limitações consequentes de um processo patológico. Resulta, também, da interação entre estas pessoas e as barreiras comportamentais e ambientais que impedem a sua participação plena e efetiva na sociedade, em condições de igualdade com as outras pessoas. Assim, a existência de barreiras arquitetónicas acentua a deficiência de qualquer pessoa com incapacidade/limitações e aumenta a sua vulnerabilidade.

A mesma Convenção ${ }^{(10)}$ ressalva os princípios universais da dignidade, integralidade e não discriminação, definindo as obrigações gerais dos governos relativas à integração das várias dimensões da deficiência nas suas políticas. Reafirma ainda, as obrigações específicas relativas à sensibilização da sociedade para a deficiência, ao combate aos estereótipos e à equidade de acesso das pessoas com deficiência.

Assim, deverão ser tomadas medidas efetivas e apropriadas que permitam às pessoas com incapacidade "atingirem e manterem um grau de independência máximo, plena aptidão física, mental, social e vocacional e plena inclusão e participação em todos os aspetos da vida" (artigo $26^{\circ}$ da CDPD). Esta mesma Convenção salvaguarda o direito de viver num ambiente físico acessível (artigo $\left.9^{\circ}\right)^{(10)}$. Perante estes direitos da pessoa com incapacidade, o segundo ponto do artigo $71^{\circ}$ da Constituição da República Portuguesa (CRP) vem reforçar a obrigação de uma "política nacional de prevenção e de tratamento, reabilitação e integração dos cidadãos portadores de deficiência e de apoio às suas famílias" (11).

É essencial a consciencialização das pessoas para a igualdade de direitos, sendo uma incumbência do Estado a "promoção do bem-estar e qualidade de vida da população e a igualdade real e jurídico-formal entre todos os portugueses" (alínea d) do artigo $9^{\circ}$ e artigo $13^{\circ}$ da CRP $^{(11)}$.

No que diz respeito à questão das acessibilidades, a regulação normativa através do Decreto-Lei n 123/97, visou a introdução de normas técnicas, no sentido de eliminar barreiras urbanísticas e arquitetónicas nos edifícios públicos, equipamentos coletivos e via pública $^{(12)}$.

Constatada a insuficiência das soluções propostas por esse diploma, revogou-se o referido Decreto-Lei ${ }^{(12)}$, com a criação de um novo diploma, que "define o regime da acessibilidade aos edifícios e estabelecimentos que recebem o público, via pública e edifícios habitacionais, visando a construção de um sistema global, coerente e ordenado em relação às acessibilidades", com vista a proporcionar às pessoas com mobilidade condicionada condições iguais aos demais. Importa referir a extensão do âmbito de aplicação das normas técnicas de acessibilidades aos edifícios habitacionais (acesso às habitações e seus interiores), para garantir a mobilidade sem condicionamentos $^{(1)}$.

Com a aplicação do Decreto-Lei n ${ }^{\circ}$ 163/06, era expectável que em fevereiro de 2017, data de término do prazo para adaptação dos espaços (edifícios, estabelecimentos e equipamentos de utilização pública cuja data de construção era anterior a 1997), não existissem barreiras arquitetónicas em locais de uso público. Contudo, a evidência demonstra que ainda subsistem barreiras arquitetónicas ${ }^{(9)}$.

Tanto a nível europeu como nacional, têm sido desenvolvidas estratégias para a promoção da acessibilidade arquitetónica, o que está implícito numa das propostas do XXI Governo Constitucional que passa pelo desenvolvimento do programa "Territórios Inclusivos" (3).

A União Europeia (UE), tem desenvolvido ações para a promoção, por exemplo, de serviços de turismo acessíveis, onde destacamos o projeto pioneiro em Portugal: Lousã - Destino de Turismo Acessível ${ }^{(13)}$.

Com o intuito de melhorar a mobilidade e acessibilidade de todos, Portugal pretende, até 2020 combater esta forma de descidadania (Plano de Ação e Mobilidade Urbana e Sustentável - PAMUS). Reconhecendo ainda a importância do conhecimento sobre as necessidades das pessoas com mobilidade condicionada, a Comissão Portuguesa para Normalização no domínio do Turismo (Subcomissão 8 - Turismo Acessível) validou um o Guia de Boas Práticas de Acessibilidade na Hotelaria com o objetivo de promover a qualidade no âmbito da acessibilidade dos serviços turísticos ${ }^{(13)}$.

Anualmente comemora-se o Dia Internacional da Pessoa com Deficiência e em 2018, o tema foi o Empoderamento de pessoas com deficiência e a garantia de inclusão e igualdade. Nesta comemoração foi, também, dada visibilidade ao décimo primeiro objetivo da Agenda 2030 - 17 Objetivos de Desenvolvimento Sustentável - cidades e comunidades inclusivas, seguras, resilientes e sustentáveis ${ }^{(14)}$. Este consiste em "proporcionar o acesso a sistemas de transporte seguros, acessíveis, sustentáveis e a preço acessivel para todos, melhorando a segurança rodoviária através da expansão da rede de transportes públicos, com especial atenção para as necessidades das pessoas em situação de vulnerabilidade, mulheres, crianças, pessoas com deficiência e idosos; aumentar a urbanização inclusiva e sustentável, e as capacidades para o planeamento e gestão das condições habitacionais participativas, integradas e sustentáveis, em todos os países; proporcionar o acesso universal a espaços públicos seguros, inclusivos, acessíveis e verdes, particularmente para as mulheres e crianças, pessoas idosas e pessoas com deficiência" (14).

A promoção da acessibilidade é fundamental na qualidade de vida das pessoas e imprescindível para o exercício da igualdade de direitos de qualquer membro de uma sociedade democrática. Desta forma e como enunciado no Decreto-Lei n. ${ }^{\circ} 163 / 06$, contribui para 
"um maior reforço dos laços sociais, para uma maior participação cívica de todos aqueles que a integram e, consequentemente, para um crescente aprofundamento da solidariedade no Estado social de direito" (1).

Neste Decreto-Lei(1), está também implícito que a abertura de estabelecimentos destinados ao público, sendo eles escolas, estabelecimentos de saúde ou comerciais, entre outros, é licenciada pelas entidades competentes, quando estes estão conforme as normas de acessibilidade. Sempre que se verifiquem situações desconformes, que não respeitem as condiçõos de acessibilidade exigíveis, estas têm que ser, obrigatoriamente, comunicadas às entidades competentes para os licenciamentos por parte da Câmara Municipal, de forma a serem indeferidos.

A acessibilidade deverá ser inclusiva e universal, para que as cidades permitam, cada vez mais, o acesso dos cidadãos, não só pelos espaços físicos que estas oferecem, mas também, pelo espaço público que deve ser livre de obstáculos materiais.

Uma maior qualidade de vida das pessoas com mobilidade condicionada, apresenta-se como uma obrigação cívica de todos os cidadãos, na melhoria das suas responsabilidades. Sabendo que as dificuldades de mobilidade afetam um conjunto vasto de pessoas, $60 \%$ nos países da $\mathrm{OCDE}^{(15)}$, estão impedidos de participar na vida económica, social e cultural, devido à presença de barreiras, tornando-se emergente adotar soluções técnicas que garantam o acesso aos edifícios, bem como a todos os espaços públicos e transportes.

As autarquias locais têm um importante papel na eliminação de barreiras, promovendo um ambiente acessível e livre de barreiras arquitetónicas, devendo aumentar a sua atuação e deixando de se limitar aos domínios das infraestruturas e dos equipamentos básicos ${ }^{(16)}$. É assim fulcral criar e ou reforçar sinergias entre as entidades públicas e privadas e o setor da saúde, num binómio de promoção da saúde e da acessibilidade para pessoas com mobilidade condicionada.

Na sua maioria, as entidades não correspondem às reais necessidades da sua população. O espaço público, o edificado e os transportes são projetados para o homem médio, de idade madura, com força e plena saúde, indo de acordo com a teoria de Leonardo Da Vinci.

Os profissionais de saúde necessitam de ir para além das estratégias de prevenção, devendo promover a saúde, facilitar o bem-estar e o equilíbrio do meio ambiente, que desde meados do século XIX até à atualidade, com a teoria ambientalista de Florence Nightingale, têm vindo a ser lideradas pela enfermagem $^{(17)}$.

No âmbito do exercício profissional, a utilização de um referencial teórico de enfermagem é essencial para dar sustentação e fundamentar a atuação do enfermeiro, devendo por isso alicerçar-se na melhor evidência científica disponível(18). De entre os referenciais teóricos verificamos que já há dois séculos que esta ciência se interroga sobre as questões ambientais.
A evidência demonstra que os Enfermeiros Especialistas em Enfermagem de Reabilitação identificam as conceções de Afaf Meleis, Dorothea Orem e Callista Roy como as que mais sustentam a sua prática ${ }^{(19)}$.

Os enfermeiros podem constituir-se como facilitadores nos processos de transição se a sua prática for centrada na pessoa e nas suas reais necessidades ${ }^{(20)}$. A transição para a dependência no autocuidado é um fator transformável através da melhoria do potencial de aprendizagem da pessoa. Os enfermeiros podem e devem contribuir de forma significativa quer na promoção do autocuidado, quer na qualidade dos processos de transição vividos pelas pessoas, através de medidas contínuas em que a equipa multidisciplinar deve estar focada e, onde o EEER pode constituir uma mais valia, sendo facilitador deste processo de transição bem sucedido.

A teoria das transições de Afaf Meleis, defende que os indivíduos passam por transições durante o seu ciclo vital, e que o enfermeiro deve ser capaz de reconhecer esses momentos e torná-los mais saudáveis(20). Os recursos da comunidade e da sociedade são condicionantes do processo de transição podendo ser facilitadores ou inibidores. Cabe assim ao EEER promover os facilitadores e diminuir ou eliminar os inibidores, nomeadamente no que se refere às barreiras arquitetónicas e à utilização de estratégias adaptativas que minimizem as mesmas.

Para Orem, o ambiente é uma das condições que afeta o autocuidado, ou seja, todas as atividades de vida diárias que são executadas pela própria pessoa para a manutenção da sua vida e do seu bem-estar(21). 0 ambiente refere-se então aos fatores externos que afetam o autocuidado; engloba aspectos físicos, químicos e biológicos englobando a família, a cultura e a comunidade.

A capacidade da pessoa se envolver e ajustar ao autocuidado, denomina-se ação de autocuidado, sendo que esta, pode ser afetada por fatores condicionantes básicos (outro dos conceitos descritos por Orem), de que são exemplo a idade, o sexo, o estado de desenvolvimento, o estado de saúde, fatores ambientais (existência de barreiras arquitectónicas), estado sociocultural, aspetos relativos à família e disponibilidade e adequação de recursos.

O modelo concetual de adaptação de Roy define o indivíduo como um ser adaptativo, sendo competência do enfermeiro facilitar o acesso a respostas adaptativas saudáveis ${ }^{(22)}$. Este referencial teórico indica que o estímulo é identificado como o elemento que desencadeia a resposta, podendo este ser intrínseco ou extrínseco à pessoa, e inclui nele todas as condições, circunstâncias e influências em volta desta, ou que interfere com o desenvolvimento ou comportamento da mesma. O termo "ambiente", neste referencial, define o conjunto de estímulos que interagem com a pessoa. Estes estímulos podem ser divididos em focal, contextual e residual ${ }^{(23)}$.

O estímulo focal é o mais relevante, pois confronta diretamente com a pessoa determinando mudanças; o estímulo contextual é o que advêm do meio interno ou externo à pessoa, com influência positiva ou negativa 
sobre a sua situação, contribuíndo para o comportamento provocado pelo estímulo focal; e os estímulos residuais são fatores ambientais internos ou externos, que têm um efeito indeterminado no comportamento da pessoa(22).

Assim, verificamos a relação entre o ambiente e o comportamento das pessoas no seu quotidiano, o seu envolvimento na comunidade e a sua integração social(21-22). Denota-se, portanto, o contributo dos referenciais teóricos na intervenção do EEER no sentido da eliminação de barreiras arquitetónicas fomentando ambientes inclusivos para pessoas com mobilidade condicionada.

Paralelamente aos referenciais teóricos, a prática de enfermagem sustenta-se nos documentos reguladores do exercício profissional. No âmbito da especialização em Enfermagem de Reabilitação, o regulamento das competências específicas do EEER acrescenta a inclusão social à promoção de saúde já contemplada nas competências do enfermeiro de cuidados gerais.

Neste seguimento, as acessibilidades constituem-se como relevantes no âmbito dos Cuidados Especializados em Enfermagem de Reabilitação, estando contemplado no Regulamento de Competências Específicas do Enfermeiro Especialista em Enfermagem de Reabilitação, que este profissional "capacita a pessoa com deficiência, limitação da atividade e ou restrição da participação para a reinserção e exercício da cidadania" e "promove a mobilidade, a acessibilidade e a participação social" pelos conhecimentos sobre legislação específica, sensibilização da comunidade no sentido de adotarem práticas inclusivas, identificação e eliminação de barreiras arquitetónicas, podendo também emitir pareceres técnico-científicos acerca de estruturas e equipamentos sociais da comunidade ${ }^{(24)}$.

Aos EEER, tal como descrito no Regulamento dos Padrões de Qualidade dos Cuidados Especializados em Enfermagem de Reabilitação, compete desenvolver processos de promoção da inclusão social das pessoas com deficiência, sendo elementos importantes: "a capacitação da comunidade para o respeito e integração da pessoa com deficiência"; "a otimização dos recursos do cliente, família e comunidade para manter e/ou promover a inclusão da pessoa com deficiência, promovendo a sua participação na vida da comunidade"; "a adoção de estratégias de discriminação positiva para os clientes mais fragilizados"; "o envolvimento do cliente, família e comunidade nas estratégias promotoras da inclusão"; "o desenvolvimento de estratégias promotoras da inclusão ativa de pessoas com deficiência, incluindo as condições de habitação, a melhoria de acesso ao emprego, formação e oportunidades de educação"; “o desenvolvimento de campanhas anti-estigma e atividades nos meios de comunicação social, escolas, empregos, ou outros contextos, de modo a promover a integração de pessoas com necessidades especiais" (25).

A ideia dos enfermeiros como líderes de estratégias de promoção da saúde também tem vindo a ser reforçada pela Organização Mundial de Saúde, que enfatiza o empoderamento da enfermagem para que se consiga conquistar a saúde e o bem-estar da população mundial, através de estratégias de promoção da saúde física, mental e do bem-estar, coordenadas por enfermeiros que tenham na sua formação um conhecimento científico capaz de lhes permitir realizar essas estratégias ${ }^{(26)}$.

Alguns autores ${ }^{(3)}$ referem que se reconhece a necessidade de formação sobre a temática da acessibilidade, sendo uma potencialidade a considerar pelas entidades públicas e privadas. Defendem ainda, que o EEER, dado possuír conhecimentos técnicocientíficos, pode sensibilizar para o impacto das barreiras arquitetónicas na qualidade de vida das pessoas com mobilidade condicionada, barreiras estas que, muitas vezes, podem ser eliminadas com medidas simples e um correto planeamento de recursos.

Os EEER marcam diferença no que diz respeito ao conhecimento da legislação específica para a acessibilidade, ao saber a quem se dirigir caso necessite de solicitar intervenção para eliminar barreiras arquitetónicas para pessoas com mobilidade condicionada na comunidade, bem como ao conhecimento do processo de ativação dos meios responsáveis para eliminar as barreiras arquitetónicas. Contudo, tal como confirmado por vários autores ${ }^{(9)}$, estes profissionais não diferem dos outros no que diz respeito ao desenvolvimento de algum tipo de intervenção nesse sentido, na sua prática profissional.

Importa, no entanto, ter em consideração que os EEER deparam-se atualmente com novos desafios dada a evolução da sociedade, cada vez mais complexa e multidimensional ${ }^{(3)}$.

Atendendo à formação e às competências já anteriormente mencionadas, cabe ao EEER promover o desenvolvimento de políticas de saúde fortes, influenciando decisões políticas, técnicas e sociais para a promoção de ambientes acessiveis, particularmente através da sensibilização e formação dos autarcas para a relevância da eliminação de barreiras arquitetónicas e para o respeito e valorização da diversidade humana, para uma inteira participação social das pessoas com mobilidade condicionada. Neste contexto, as entidades públicas e privadas são decisivas para a construção de territórios inclusivos para estas pessoas.

Uma das intervenções do EEER, indo de encontro às políticas de saúde em vigor, é a sua contribuição para o aumento da literacia em saúde da população e, neste caso específico, da pessoa com mobilidade condicionada e do seu familiar cuidador. Quanto maior o conhecimento que estes possuem em relação ao seu estado de saúde e aos recursos existentes na comunidade, maior será a sua autonomia, autogestão da doença, autoeficácia, uso assertivo dos serviços de saúde e empoderamento ${ }^{(27)}$.

Sendo um dos principais objetivos da enfermagem a obtenção de ganhos em saúde da pessoa, um aumento da literacia é indispensável, visto esta estar associada tanto direta, como indiretamente a maior outcome em saúde ${ }^{(28)}$.

O empoderamento da pessoa com mobilidade condicionada corrobora com a centralização na pessoa, responsabilizando-a pelos cuidados de saúde que aufere 
e na identificação de condicionantes dificultadoras no seu quotidiano e na promoção de políticas inclusivas. A pessoa com mobilidade condicionada poderá ser um agente passivo ou ativo, sendo importante a sua participação ativa, colocando-a ao nível da tomada de decisão política, aspeto que deverá ser impulsionado pelo EEER.

\section{CONCLUSÃO}

Embora ainda exista uma significativa oportunidade de melhoria, a temática da acessibilidade tem vindo a assumir um papel cada vez mais relevante na atuação do EEER no âmbito de entidades públicas e privadas, em resultado de um despertar de consciências cada vez mais visível.

As barreiras à acessibilidade prejudicam a igualdade de oportunidades, favorecem a discriminação, acentuam preconceitos e aumentam a deficiência da pessoa, aspetos mais que suficientes, para exigirem uma intervenção sistematizada dos profissionais.

A promoção da acessibilidade constitui um elemento fundamental na qualidade de vida das pessoas, sendo um meio imprescindível para o exercício dos direitos que são conferidos a qualquer membro de uma sociedade democrática, aspeto que reforça, mais uma vez, a necessidade dos EEER considerarem esta componente no âmbito da conceção e prestação dos cuidados de enfermagem de reabilitação.

Para a melhoria da acessibilidade é crucial uma tomada de decisão centrada no processo de enfermagem que evidencie um olhar holístico e potencialmente empoderador quer da pessoa com mobilidade condicionada e do seu familiar cuidador, quer da comunidade e dos stakeholders, e, particularmente dos enfermeiros especialistas em enfermagem de reabilitação.

Enquanto conhecedor de leis e do processo de reabilitação, o EEER deverá ainda intervir no sentido de aumentar a literacia das pessoas com incapacidade e da sua família sobre a sua condição, de modo a promover a sua autonomia e independência. O empoderamento das pessoas com incapacidade tem vindo a ser o lema.

A enfermagem enquanto disciplina científica tem como alvo, não só a pessoa, mas também, a família, a comunidade ou a sociedade. Portanto, o EEER é o impulsionador e o condutor para a integração da pessoa no seu meio, intervindo na sua autonomia e na adaptação ao ambiente, de maneira a que as suas limitações não impeçam o exercício da cidadania.

Este artigo teórico evidencia-se como relevante, enquadrando de forma objetiva o contributo dos EEER como agentes preponderantes e participantes ativos, quer no processo de tomada de decisão político-social, quer no sentido de facilitar o acesso a respostas adaptativas saudáveis da pessoa com mobilidade condicionada.

No futuro, tendo em conta que esta temática terá ainda maior pertinência, deveria ser-lhe atribuído maior valor na formação do EEER e dos restantes atores, analisando-se o seu contributo na definição das políticas sociais e no outcome adaptativo da pessoa com mobilidade condicionada em todos os seus contextos.

\section{REFERÊNCIAS BIBLIOGRÁFICAS}

1. Decreto-Lei n. ${ }^{\circ} 163 / 06$ de 8 de Agosto. Diário da República n. ${ }^{\circ}$ 152/16, I Série. Ministério do Trabalho e da Solidariedade Social. Lisboa, Portugal. 2016.

2. Organização Mundial da Saúde. Classificação Internacional de Funcionalidade, Incapacidade e Saúde. Lisboa: Direção Geral da Saúde. 2004

3. Pereira R, Gomes M, Schoeller S, Aguilera J, Ribeiro I, Cunha P (2018). As autarquias e a promoção da acessibilidade arquitetónica. Rev Enf Ref. 2018; IV ${ }^{(18)}: 29-38$. Disponível em https://doi.org/10.12707/RIV18022.

4. Organização Mundial da Saúde. Relatório mundial sobre a deficiência. São Paulo. 2011. Disponível em http: //www.pessoacomdeficiencia.sp.gov.br/usr/share/documents/ RELATORIO_MUNDIAL_COMPLETO.pdf.

5. Nações Unidas. Department of Economic and Social Affairs, Population Division. World Population Prospects. 2017. Disponível em https://esa.un.org/unpd/wpp/publications/files/wpp2017_keyfindi ngs.pdf.

6. Instituto Nacional de Estatística IP. Censos 2011 Resultados Definitivos - Portugal. Lisboa. 2012. Disponível em https://censos.ine.pt/ngt_server/attachfileu.jsp?look_parentBoui=1 48313382\&att_display=n\&att_download=y.

7. Martins J. Seminário: Investigação em segurança e saúde ocupacionais presente e futuro. Universidade do Porto. 2011.

8. Ramos $C$ in Nota Introdutória. Acessibilidade e Mobilidade para todos - Apontamentos para uma melhor interpretação do DL n. ${ }^{\circ}$ 163/2006 de 8 de Agosto. Porto: Inova. 2007.

9. Pereira RSS, Martins MM, Gomes B, Aguilera JAL, Santos J. A intervenção do Enfermeiro de Reabilitação na promoção da Acessibilidade. Rev Port de Enferm de Reabil. 2018; ${ }^{(2)}: 66-72$. Disponível

https: / /www.aper.pt/Ficheiros/Revista/RPERV1N2.pdf.

10. Alves $\mathrm{C}$ et al. Direitos das Pessoas com Deficiência. Lisboa, Portugal. 2017. Disponível em http://www.cej.mj.pt/cej/recursos/ebooks/civil/eb_DireitoPessoas D2017.pdf.

11. Constituição da República Portuguesa. VII Revisão constitucional. $2005 . \quad$ Disponível em https: / / www.parlamento.pt/Legislacao/Paginas/ConstituicaoRepubl icaPortugu esa.aspx.

12. Decreto-Lei n. ${ }^{\circ} 123 / 97$ de 22 de Maio. Diário da República n. ${ }^{\circ} 118$. I Série-A. Ministério da Solidadriedade e Segurança Social. Lisboa, Portugal. 1997.

13. Devile E, Garcia A, Carvalho F \& Neves J. Turismo Acessível em Portugal - Estudo de casos de boas práticas. Rev Tur Desenvolv. 2012; $17^{(18)}: 1403-1416$.

14. Centro de Informação Regional das Nações Unidas para a Europa Ocidental. Agenda 2030 de Desenvolvimento Sustentável. 2016. Disponível

https://www.unric.org/pt/images/stories/2016/ods_2edicao_web_ pages.pdf.

15. Teles P. A acessibilidade universal na qualificação social e física das cidades - das cidades e vilas com mobilidade para todos ao Portugal 2020. 2017. Omnia (6). Disponível em http://mobilidadept.com/_upl/files/paula-teles-omnia-

170523130255.pdf.

16. Falcato J. Autarquias e inclusão das pessoas com deficiência. 2017. Disponível em https://www.esquerda.net/artigo/autarquiase-inclusao-das-pessoas-com-deficiencia/50202.

17. Medeiros A, Enders B \& Lira A. Teoria Ambientalista de Florence Nightingale: Uma Análise Crítica. Esc Anna Nery Rev Enferm. 2015; 19(3):518-524 Recuperado http: //www.scielo.br/pdf/ean/v19n3/1414-8145-ean-19-03-0518.

18. Ribeiro O, Martins M, Tronchin D, Silva J. Exercício profissional dos enfermeiros sustentado nos referenciais teóricos da disciplina: realidade ou utopia. Revista de Enfermagem Referência. 2018. $4^{(19)}: 39-48$

19. Martins M, Ribeiro O \& Silva J. Orientações concetuais dos 
Enfermeiros Especialistas em Enfermagem de Reabilitação em hospitais portugueses. Rev Port Enferm Reabil. 2018; $1^{(2)}: 43-48$.

20. Meleis $\mathrm{A}$ et al. Experiencing transitions: an emerging middle-range theory. Advances in Nursing Science. 2000. 23(1).

21. Orem D E. Normas práticas en enfermería. Madrid : Piramide. 1983. 84-368-0224-1.

22. Roy C \& Andrews H A. The Roy adaptation model. Lisboa, Portugal: Instituto Piaget. 2001.

23. Medeiros $L$ et al. Modelo de Adaptacão de Roy: revisão integrativa dos estudos realizados à luz da teoria. Revista Rene. 2015. 16 ${ }^{(1)}: 132-$ 140.

24. Regulamento n. ${ }^{\circ} 125 / 11$ de 18 de fevereiro. Diário da República n. ${ }^{\circ}$ 35/11, II Série. Ordem dos Enfermeiros. Lisboa, Portugal. 2011.

25. Regulamento $n .^{\circ} 350 / 15$ de 22 de Junho. Diário da República n. ${ }^{\circ}$ 119/15, II Série. Ordem dos Enfermeiros. Lisboa, Portugal. 2015.

26. World Health Organization. Global trategic directions for strengthening nursing and midwifery 2016-2020. 2016. Disponível em http://www.who.int/hrh/nursing_midwifery/global-strategicmidwifery2016-2020.pdf.

27. Doyle G, Cafferkey K \& Fullam J. The European Health Literacy Survey: Results from Ireland: Dublin, Ireland. University College Dublin. 2012.

28. Nutbeam D.Defining and measuring health literacy: what can we learn from literacy studiesInt J public health. 2009; 54:303-305. 